\title{
Resonance Raman spectrum of doped epitaxial graphene at the Lifshitz transition
}

\author{
Martin G. Hell, ${ }^{\dagger}$ Niels Ehlen, ${ }^{\dagger}$ Boris V. Senkovskiy, ${ }^{\dagger}$ Eddwi H. Hasdeo, ${ }^{\dagger}$ \\ Alexander Fedorov, ${ }^{\dagger}$ Daniela Dombrowski, ${ }^{\dagger}$ Carsten Busse,${ }^{\dagger}$ Thomas Michely $^{\dagger}$ \\ Giovanni di Santo,,$^{\perp}$ Luca Petaccia, ${ }^{\perp}$ Riichiro Saito ${ }^{\ddagger}$ and Alexander Grüneis*,\# \\ II. Physikalisches Institut, Universität zu Köln, Zülpicher Strasse 77, 50937 Köln, \\ Germany, Department of Physics, Tohoku University, Sendai 980-8578, Japan, Research \\ Center for Physics, Indonesian Institute of Sciences, Kawasan Puspiptek Serpong, \\ Tangerang Selatan, 15314, Indonesia, Institut für Materialphysik, Westfälische \\ Wilhelms-Universität Münster, Wilhelm-Klemm-Str. 10, 48149 Münster, Germany, \\ Fakultät IV Physik, Universität Siegen, Walter-Flex-Str. 3, 57072 Siegen, Germany, \\ Elettra Sincrotrone Trieste, Strada Statale $14 \mathrm{~km}$ 163.5, 34149 Trieste, Italy, and II. \\ Physikalisches Institut, Universität zu Köln, Zülpicher Strasse 77, 50937 Köln, Germany \\ E-mail: grueneis@ph2.uni-koeln.de
}

\footnotetext{
Abstract

${ }^{*}$ To whom correspondence should be addressed

${ }^{\dagger}$ II. Physikalisches Institut, Universität zu Köln, Zülpicher Strasse 77, 50937 Köln, Germany

$\ddagger$ Department of Physics, Tohoku University, Sendai 980-8578, Japan

ॠResearch Center for Physics, Indonesian Institute of Sciences, Kawasan Puspiptek Serpong, Tangerang Selatan, 15314, Indonesia

§Institut für Materialphysik, Westfälische Wilhelms-Universität Münster, Wilhelm-Klemm-Str. 10, 48149 Münster, Germany

"Fakultät IV Physik, Universität Siegen, Walter-Flex-Str. 3, 57072 Siegen, Germany

${ }^{\perp}$ Elettra Sincrotrone Trieste, Strada Statale $14 \mathrm{~km} \mathrm{163.5,} 34149$ Trieste, Italy

\# II. Physikalisches Institut, Universität zu Köln, Zülpicher Strasse 77, 50937 Köln, Germany
} 
We employ ultra-high vacuum (UHV) Raman spectroscopy in tandem with angleresolved photoemission (ARPES) to investigate the doping-dependent Raman spectrum of epitaxial graphene on $\operatorname{Ir}(111)$. The evolution of Raman spectra from pristine to heavily Cs doped graphene up to a carrier concentration of $4.4 \times 10^{14} \mathrm{~cm}^{-2}$ is investigated. At this doping graphene is at the onset of the Lifshitz transition and renormalization effects reduce the electronic bandwidth. The optical transition at the saddle point in the Brillouin zone then becomes experimentally accessible by ultraviolet (UV) light excitation which achieves resonance Raman conditions in close vicinity to the van Hove singularity in the joint density of states. The position of the Raman $G$ band of fully doped graphene/Ir(111) shifts down by $\sim 60 \mathrm{~cm}^{-1}$. The $G$ band asymmetry of Cs doped epitaxial graphene assumes an unusual strong Fano asymmetry opposite to that of the $G$ band of doped graphene on insulators. Our calculations can fully explain these observations by substrate dependent quantum interference effects in the scattering pathways for vibrational and electronic Raman scattering.

Keywords: alkali doping, graphene, UHV Raman, ARPES, Lifshitz

\section{Introduction}

Raman spectroscopy is the most widely used characterization method for graphene. ${ }^{1-4}$ The electron and phonon systems of graphene are strongly coupled to each other by electronphonon interactions. ${ }^{5,6}$ These interactions manifest as "kink" features in the electronic spectral function ${ }^{7-12}$ and as Kohn anomalies in the phonon dispersion relations around the Bril-

louin zone $(\mathrm{BZ})$ center ( $\Gamma$ point) and corners $\left(K\right.$ points). ${ }^{5,6}$ As a consequence, the carrier concentration of graphene sensitively affects the position, line shape and intensity of the first- and second order Raman spectra corresponding to these phonon modes. The effects of phonon renormalization due to the removal of the Kohn anomaly or lattice expansion on the phonon energy are quantitatively understood. ${ }^{5}$ Phonon renormalization results in a 
phonon upshift for Fermi level positions higher than half the phonon frequency (measured from the Dirac point) and lattice expansion results in a phonon downshift. ${ }^{5,6}$ Experimentally, phonon hardening has been observed in Raman measurements where the charge carrier concentration of graphene has been tuned by field effect gating ${ }^{6,13}$ or by ionic liquid gating. ${ }^{14-16}$ The latter approach has been used to induce large carrier densities of $6 \times 10^{13} \mathrm{~cm}^{-2}$ (Ref. 15). Even for such high carrier concentrations, phonon hardening due to phonon self-energy corrections dominates. ${ }^{5}$ However, when the carrier density is in the $10^{14} \mathrm{~cm}^{-2}$ range that has already been probed by transport ${ }^{17,18}$, the Fermi energy can be in the vicinity of the van Hove singular energy at the $M$ point in the Brillouin zone and phonon softening will then dominate. Achieving and probing high carrier densities is fundamentally important for both conventional ${ }^{19-21}$ and chiral superconductivity ${ }^{22}$ in monolayer graphene. The latter case requires the Fermi level to touch the saddle point van Hove singularity at the $M$ point in the two-dimensional (2D) BZ. In this case, the Fermi surface of graphene assumes a closed shape centered at the $\Gamma$ point rather than two surfaces centered around the $K$ and $K^{\prime}$ points. The transition of topology from two Fermi surfaces to one Fermi surface marks the Lifshitz transition ${ }^{23}$ in graphene.

The present work aims at understanding the peculiar Raman spectrum of graphene at the Lifshitz transition and unravelling the deep underlying connection between electronic band structure and phonon renormalization in heavily doped graphene. To that end we synthesize Cs doped graphene up to the highest achievable carrier concentrations. The energy bands are renormalized significantly due to doping and are probed by angle-resolved photoemission spectroscopy (ARPES) for each doping step. Despite Raman spectroscopy is typically not considered a surface science method, we present an original ultra-high-vacuum (UHV) Raman setup empoloying a commercial Raman system that is coupled to a UHV system. From these experiments we can relate the observed changes in the vibrational and electronic spectrum to band structure changes. We thus obtain a complete picture of the coupled 
electron-phonon system in epitaxial graphene.

McChesney et al. already experimentally observed the Lifshitz transition in heavily doped graphene using ARPES. ${ }^{24}$ Their key finding was that the band structure of heavily doped graphene is strongly renormalized, yielding a flat conduction band at the Fermi level, i.e. an extended van Hove singularity. ${ }^{24}$ Importantly, the renormalization also reduces the transition energy at the saddle point between valence and conduction bands. Indeed, Mak et al. found that the energy of the $M$ point transition is reduced by $\sim 200 \mathrm{meV}$ when going from charge neutrality to $1 \times 10^{14}$ carriers per $\mathrm{cm}^{2}$ (Ref. 25).

For graphene, the first-order Raman spectrum due to zone-center optical phonons with in-plane polarization (the $G$ band) shows an asymmetric Fano lineshape if graphene is doped by field effect gating ${ }^{26}$ or alkali metal doping ${ }^{27}$. This lineshape is reproduced theoretically by considering the interference between a discrete transition (phonon) to a continuum (excitation of electron-hole pairs) which is known as electronic Raman spectra (ERS). ${ }^{28-31}$ The Fano lineshape of the $G$ band is more pronounced for larger doping levels ${ }^{26}$ and for a higher layer number. ${ }^{27}$ The most pronounced Fano asymmetries are obtained in stage 1 graphite intercalation compounds such as $\mathrm{KC}_{8} \cdot{ }^{32,33}$ Doping also has a strong influence on the $G$ band intensity ${ }^{34}$ which is determined by quantum interference in the Raman scattering pathways. ${ }^{15,35}$ Upon doping, transitions between two occupied (unoccupied) states are forbidden by Pauli blocking and do not contribute to the total Raman intensity. As a consequence, the $G$ band intensity as a function of doping level is peaked for the condition $2 E_{F}=E_{\text {laser }}-E_{p h} / 2$. Here $E_{F}$ is the Fermi level position measured from the charge neutrality point, $E_{\text {laser }}$ the excitation energy and $E_{p h}$ the $G$ band phonon energy. ${ }^{15}$

Apart from the limits regarding carrier concentration, ionic liquid gating experiments also block the direct access to the sample surface. This precludes direct observation of the electron energy band structure of gated graphene by ARPES. It thus prohibits a detailed understanding of the non-rigid band shifting and electron-phonon coupling in heavily doped 
graphene that has been achieved for chemically doped graphene. ${ }^{7-12,24}$ An approach to carry out Raman spectroscopy of chemically doped graphene is to measure it inside a quartz ampoule. For example, alkali metal doped graphene ${ }^{27,36,37}$ and $\mathrm{FeCl}_{3}$ doped graphene ${ }^{38}$ have been measured in this way. Unfortunately, such experimental setups also preclude band structure measurements by ARPES, structural characterization by low energy electron diffraction (LEED) and efficient sample cooling to liquid He temperatures. Moreover, in the case of alkali doping inside quartz ampoules, a fraction of the dopant atoms will be oxidized because of poor vacuum conditions. Combined ARPES and Raman experiments in UHV conditions would not suffer from these drawbacks. Previously, the investigation of the electronic structure of doped graphene has been performed using the standard surface science methods such as ARPES ${ }^{9,39,40}$ and scanning tunneling spectroscopy (STS) ${ }^{21,41}$. These probe the electronic structure of epitaxially grown graphene and directly reveal Fermi level shifts, spectral functions and superconducting gaps as a function of dopant concentration and type and substrate interactions. Much less is known about the phonons and low energy electronic excitations in alkali metal doped epitaxial graphene despite these contain valuable information regarding doping level, strain and electron-phonon coupling.

\section{Experimental Results}

\section{Electronic structure of Cs doped graphene/Ir(111)}

In Figure 1a we show ARPES spectra of pristine and Cs doped graphene. For each amount of deposited Cs, we also have performed structural characterization by LEED (see supplementary information). Pristine graphene on $\operatorname{Ir}(111)$ (see Methods section for details pertaining synthesis) has the R0 structural phase. ${ }^{42}$ This is confirmed by the moiré pattern observed in LEED. The moiré pattern is a result of corrugations due to chemically modulated substrate interaction. ${ }^{43}$ It hosts weakly covalently bonded regions with a small charge transfer from graphene to $\operatorname{Ir}(111) .{ }^{43}$ Using ARPES (Figure 1a) we find in agreement to previous 

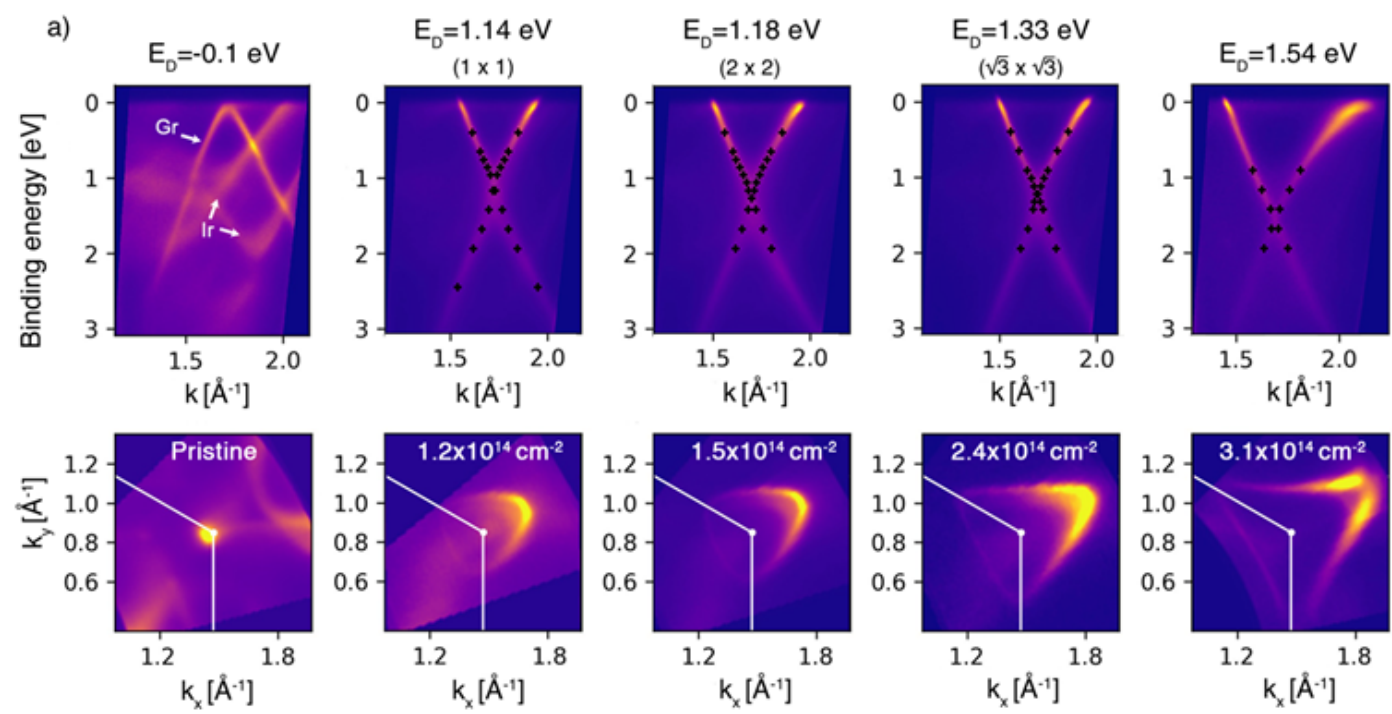

b)
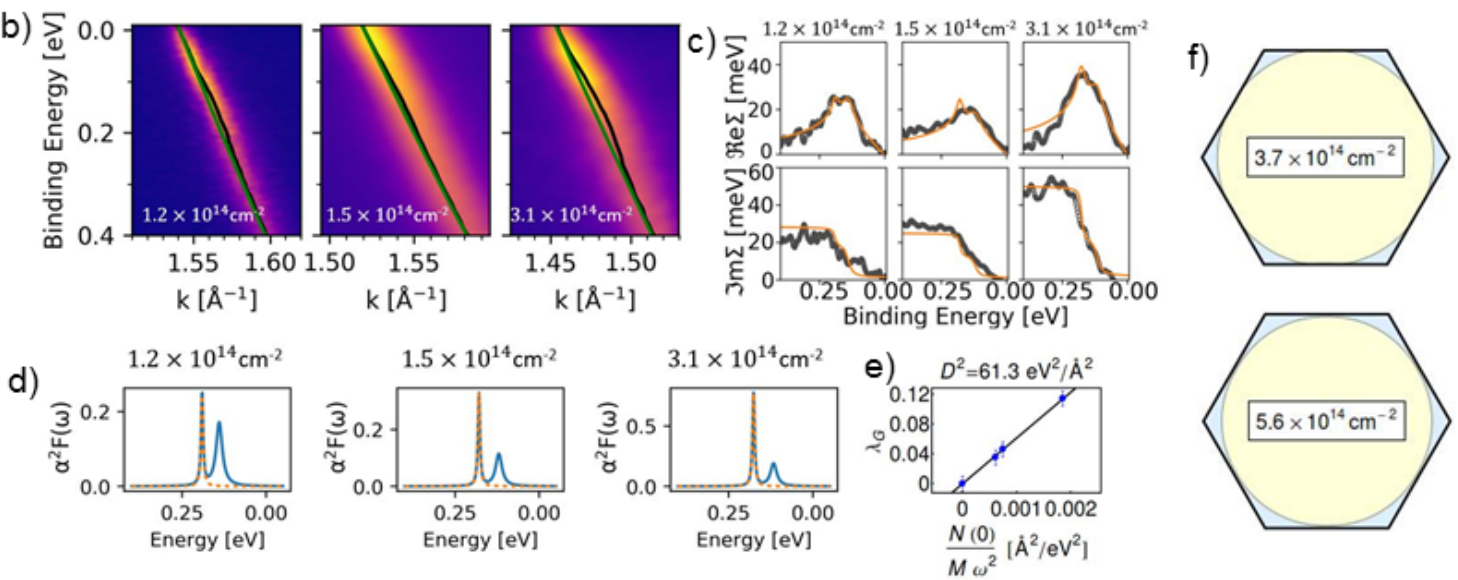

Figure 1: (a) ARPES scans of Cs doped graphene/Ir(111) in the $\Gamma K M$ direction (top panel) and Fermi surface maps (bottom panel) for different Cs coverages. The shift of the Dirac point is indicated in the top panel and the carrier concentration of graphene per $\mathrm{cm}^{2}$ is indicated in the bottom panel. (b) High-resolution ARPES data in the vicinity of the kink feature along the $\Gamma K$ direction. Black and green lines denote ARPES intensity maxima and the bare band, respectively. (c) Real and imaginary part of the self-energy (denoted as $\mathfrak{R e} \Sigma$ and $\mathfrak{I m} \Sigma$ ) for different doping levels. (d) Eliashberg functions (blue) and contribution of the $G$ band phonon (orange) along $\Gamma K$ as a function of carrier concentration. The orange lines in panel (c) denote the self-energy functions that are calculated from these Eliashberg functions. (e) Fit of the deformation potential $D^{2}$ (see text for details). (f) Tight-binding calculation of the Fermi surfaces of doped graphene (blue regions) for carrier concentrations just before and beyond the Lifshitz transition. 
literature $^{42,44}$ that $R 0$ graphene/Ir(111) is only weakly hole doped. After depositing Cs onto the sample surface at room temperature, we find that the moiré pattern observed in LEED disappears and only the first order diffraction spots are left. This LEED pattern is denoted as $1 \times 1$ in the following. The disappearance of the moiré pattern upon Cs deposition is an indication of a change in the graphene-substrate interaction. The graphene- $\operatorname{Ir}(111)$ interaction also manifests in minigaps ${ }^{44}$ which are visible in the ARPES spectrum as regions of weaker $\pi$ band intensity. After Cs deposition, these minigaps disappear, indicating that Cs doping weakens the local variations in the graphene-Ir(111) interaction. We expect that the charge transfer to graphene becomes homogeneous and removes the hybridization of $\mathrm{C}$ and Ir bands. ARPES also confirms a single doping phase as only one Dirac cone is visible. Increasing the Cs amount, we are able to reach an ordered $2 \times 2$ phase of Cs on graphene as reported previously. ${ }^{45}$ This phase also has a single Dirac cone in ARPES. Analysis of the experimental Fermi surface from ARPES measurements yields a carrier concentration of $n=1.5 \times 10^{14} \mathrm{~cm}^{-2}$. Further increasing Cs deposition leads to the $\sqrt{3} \times \sqrt{3}$ phase in LEED and a slightly higher doping level. Here we have $n=2.4 \times 10^{14} \mathrm{~cm}^{-2}$. In order to confirm the ARPES derived carrier densities by another method, we also performed Fourier transform scanning tunneling spectroscopy (FT-STS) measurements ${ }^{41}$ (see supporting information). These indicate carrier concentrations of $1.7 \times 10^{14} \mathrm{~cm}^{-2}$ for the $2 \times 2$ phase and $1.9 \times 10^{14} \mathrm{~cm}^{-2}$ for the $\sqrt{3} \times \sqrt{3}$ phase. The carrier concentration for the $2 \times 2$ is in excellent agreement to the value from ARPES and to previous experiments ${ }^{45}$. However, the concentration for the $\sqrt{3} \times \sqrt{3}$ phase from FT-STS is lower by a factor $\sim 1.3$ when compared to ARPES. This can be understood by the $\sqrt{3} \times \sqrt{3}$ phase corresponding to graphene fully intercalated with Cs. Any extra Cs lies on top of graphene and a system with small amounts of extra Cs on top still shows a $\sqrt{3} \times \sqrt{3}$ diffraction pattern in LEED. Thus the $\sqrt{3} \times \sqrt{3}$ phase exists for a broader range of adsorbate concentrations and can slightly vary from system to system. Thus LEED is a good measure of stoichiometry for the $2 \times 2$ phase only. In order to ensure depositing of equal Cs amounts in the Raman and ARPES investigations, 
we calibrated the deposited Cs using a quartz microbalance as a multiple of the amount of Cs needed for reaching the $2 \times 2$ phase. Since the $2 \times 2$ phase is well defined, this approach yields reproducible sample stoichiometries for different samples and in different experimental setups.

a)

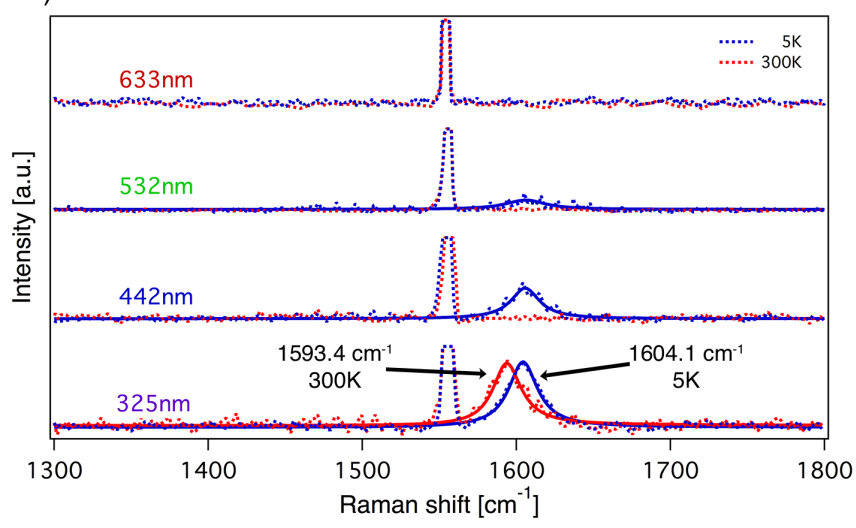

b)

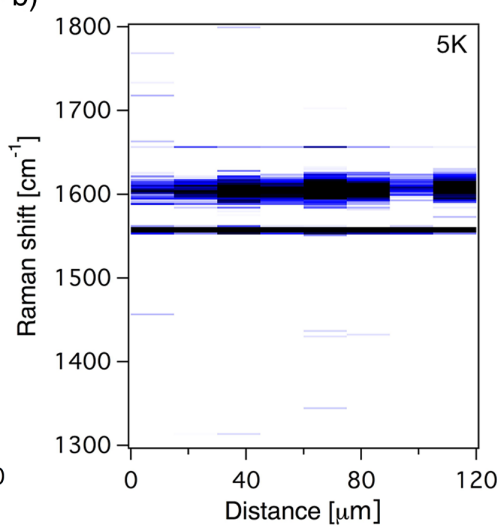

Figure 2: (a) Ultra-high vacuum (UHV) Raman spectrum of pristine graphene/Ir(111) at $T=5 \mathrm{~K}$ and $T=300 \mathrm{~K}$ for four different laser wavelengths in the range between red and ultraviolet. The dashed lines denote the experimental data and solid lines Lorentzian fits. The $G$ band taken at $325 \mathrm{~nm}$ excitation shows an upshift in frequency upon cooling (indicated by arrows in the lower panel). The sharp line at $1555 \mathrm{~cm}^{-1}$ is due to oxygen in the unavoidable part of the laser path outside the vacuum (see supporting information Figure S1 for a sketch of the UHV Raman setup). (b) Linescan of the Raman spectrum (at $325 \mathrm{~nm}$ ) across a $120 \mu \mathrm{m}$ distance of the sample.

Evaporation of excess Cs onto the sample does not result in an ordered phase according to LEED but it is still possible to increase the graphene doping level. At $n=3.1 \times 10^{14} \mathrm{~cm}^{-2}$ (see Figure 1a) we already see marked deviations from the usually observed trigonal warping in ARPES. As is shown in Figure 1a (bottom row), the warping direction changes from convex to concave. We are able to reach a value of $n=4.4 \times 10^{14} \mathrm{~cm}^{-2}$ (For the highest value of $n$, see supplementary information). Regarding the relation between Cs/C stoichiometry and carrier concentration, we note that each Cs atom may donate less than one electron. However, this does not affect the carrier densities determined from ARPES because this method is not dependent on the stoichiometry but directly measures the carrier concentration of graphene from the area of the Fermi surface of graphene. 
Let us now turn to the analysis of electron-phonon coupling. Figures 1b depicts high resolution scans of the "kink" feature at low binding energy. We first perform a standard self-consistent self-energy analysis ${ }^{7-9}$ of ARPES data in the kink region yielding the complex self-energy and the Eliashberg function. These are depicted in Figures 1c and 1d, respectively. By integrating the Eliashberg function of graphene ${ }^{8}$ in the region of the $G$ band, we obtain $\lambda_{G}$, the electron-phonon coupling constant for the $G$ mode that is frequently used in ARPES literature. The Raman community often expresses electron-phonon coupling by the deformation potential $D^{2}$. The deformation potential and the electron-phonon coupling constant $\lambda_{G}$ are connected as $\lambda_{G}=N(0) D^{2} /\left(M \omega^{2}\right)$ (see Ref. 19). Here $N(0)$ is the electron density of states per unit cell, per eV and per spin at the Fermi level, $M$ the carbon mass and $\omega$ the $G$ band frequency. The resulting fit of four charge carrier concentrations is depicted in Figure 1e and yields $D^{2}=61.3 \mathrm{eV}^{2} / \AA^{2}$. Finally, Figure 1f depicts a tight-binding calculation of the Fermi surfaces at carrier concentrations just before and beyond the Lifshitz transition. In this Figure we show Fermi surface contours at $n=3.7 \times 10^{14} \mathrm{~cm}^{-2}$ and at $n=5.6 \times 10^{14} \mathrm{~cm}^{-2}$. For the higher concentration of $n=5.6 \times 10^{14} \mathrm{~cm}^{-2}$, we are already above the Lifshitz transition (i.e. the Fermi surface is a single contour) while the lower concentration is just before the Lifshitz transition (i.e. the Fermi surface consists of two contours that almost touch). For the calculation of these Fermi surface contours we have employed a third-nearest-neighbor tight-binding model ${ }^{46}$ where the matrix elements are fitted to the experimental ARPES band structure. It is clear that the carrier concentration at which the Lifshitz transition happens, is in between these two concentrations. Using the same tightbinding model, we estimate a value of $n=4.4 \times 10^{14} \mathrm{~cm}^{-2}$ where the Lifshitz transition is observable by resonance Raman. We expect that the Lifshitz transition is induced purely by doping because we do not observe a lattice deformation. This is corroborated by the diffraction pattern of an overdoped sample above the $\sqrt{3} \times \sqrt{3}$ phase to a doping level close to the Lifshitz transition. The diffraction pattern of this sample [shown in the supporting information in Figure S2(e)] still shows sharp spots in a hexagonal pattern that are due to 
graphene.

\section{Raman spectrum of epitaxial graphene/Ir(111)}

In Figure 2 we show the Raman spectra at $\mathrm{T}=300 \mathrm{~K}$ and at $\mathrm{T}=5 \mathrm{~K}$ of epitaxial graphene/ $\operatorname{Ir}(111)$ measured by lasers with wavelengths $633 \mathrm{~mm}, 532 \mathrm{~nm}, 442 \mathrm{~nm}$ and $325 \mathrm{~nm}$. The depicted spectra are the average over 25 points chosen along a scan across $120 \mu m$ on the surface. In principle, for interpretation of the observed temperature-dependent spectra, in-plane strain and wrinkle formation due to the different thermal expansion coefficient of graphene and the Ir substrate must be considered. ${ }^{47-49}$ Only the ultraviolet (UV) laser $(325 \mathrm{~nm})$ results in a strong Raman signal at room temperature. Upon cooling, the spectra taken by $532 \mathrm{~nm}$ and $442 \mathrm{~nm}$ excitation show a weak Raman signal which could be a sign of temperature induced changes in the substrate interaction. These observations extend previous works reporting the absence of a Raman signal for graphene/Ir(111) that belongs to the $R 0$ structural phase

for visible excitation at room temperature. ${ }^{42}$ So far, no quantitative explanation regarding the absence of a Raman signal for visible laser excitation for $R 0$ graphene on $\operatorname{Ir}(111)$ has been given. We speculate that it could be explained in terms of minigaps ${ }^{44}$ which appear at certain energies in the band structure. It has been shown by ARPES that the minigaps are in all directions around $K$ point and close nowhere. ${ }^{50}$ If the laser energy hits a minigap, no electrons can be excited between the valence and conduction $\pi$ bands of graphene and the Raman intensity is supressed. The high quality of graphene is also corroborated by the absence of a defect related $D$ peak. Interestingly the $2 D$ peak is absent in all measurements. This observation is in agreement to previous works and might be related to the short lifetime of photoexcited charge carriers in graphene adsorbed on metals which supress the 2D intensity. ${ }^{51}$

\section{Temperature induced strain in epitaxial graphene}

Let us now move to the Raman analysis of $\operatorname{strain}^{52,53}$ induced by the temperature dependent change in the lattice constant. For epitaxial graphene on Ir, the thermal expansion of graphene essentially follows the substrate. ${ }^{54}$ The nonlinearity in $T$ of the thermal expansion 
coefficient $\alpha(T)$ of iridium must be taken into account. ${ }^{55,56}$ To accurately describe the expansion of the Ir substrate in the temperature range explored $(5 \mathrm{~K}-300 \mathrm{~K})$, we fit $\alpha(T)$ to literature values ${ }^{55,56}$ and calculate the expansion as $l^{\prime} / l=\exp \left(\int \alpha(T) d T\right)$. Here $l^{\prime}$ and $l$ are the lattice constants at temperatures corresponding to the upper and lower border of the integral, respectively. For describing the Ir lattice expansion of the present experiment, the integral above is taken from $T=5 \mathrm{~K}$ to $T=300 \mathrm{~K}$. The strain $\epsilon$ in per cent is calculated as $100\left(l^{\prime} / l-1\right)$ yielding $\epsilon=0.134 \%$. The phonon downshift corresponding to $\epsilon$ is given by $\Delta \omega=-2 \omega_{0} \gamma \epsilon$ (see Ref. 57 and references therein). Here $\gamma=1.99$ (Ref. 57) is the Grüneisen parameter of the doubly degenerate $G$ phonon mode of graphene, $\omega_{0}=1606.5 \mathrm{~cm}^{-1}$ is the phonon frequency at $5 \mathrm{~K}$. These values yield a strain induced downshift of the $\mathrm{G}$ band phonon when going from $5 \mathrm{~K}$ to $300 \mathrm{~K}$ of $\Delta \omega=-8.6 \mathrm{~cm}^{-1}$ which is in excellent agreement to the experimental value of $\Delta \omega=-10.7 \mathrm{~cm}^{-1}$.
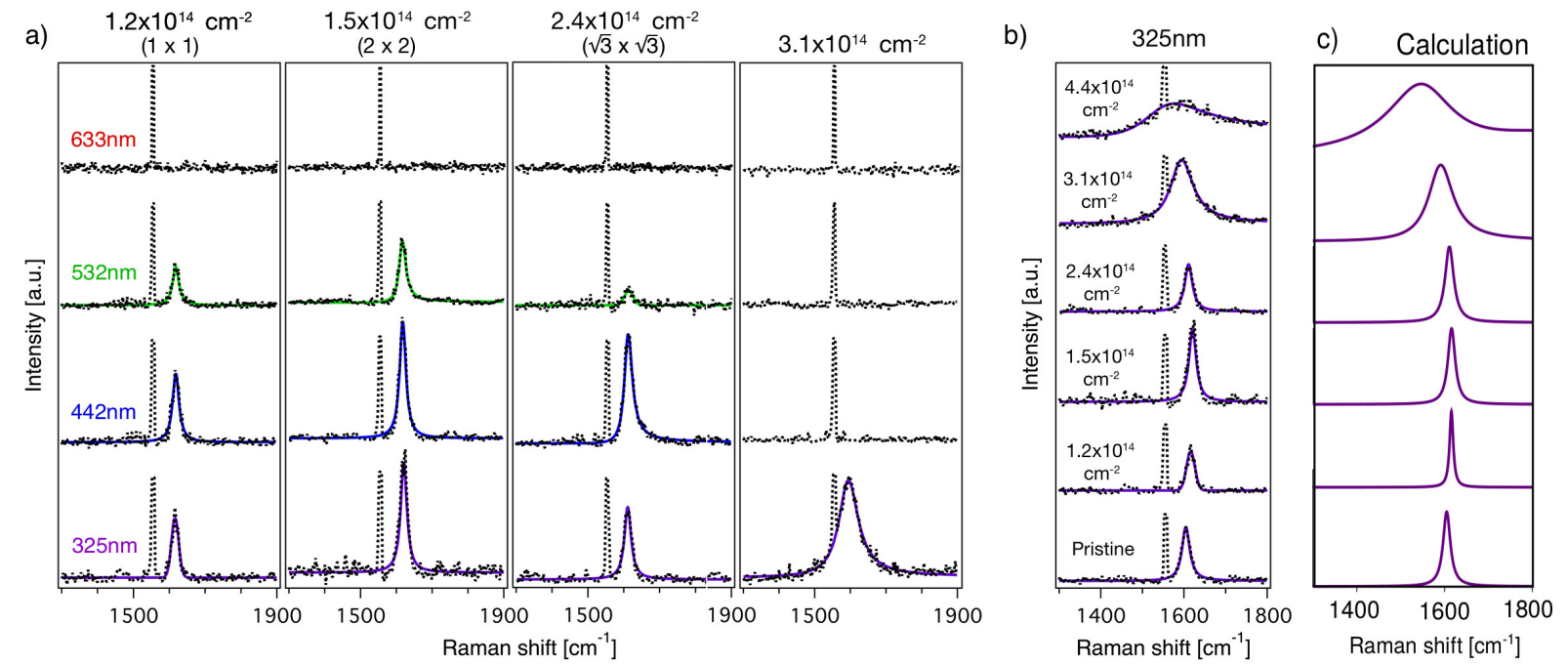

Figure 3: (a) Ultra-high vacuum (UHV) Raman spectra of Cs doped epitaxial graphene/Ir(111) with increasing carrier concentration from left to right measured by four laser lines. The raw data (dots) of the Raman $G$ band together with a Fano lineshape fit are shown. (b) UHV ultraviolet Raman spectra of doped graphene with increasing carrier concentration measured with $325 \mathrm{~nm}$ excitation. All Raman spectra are taken at $T=5 \mathrm{~K}$ and a vacuum better than $2 \times 10^{-10}$ mbar. (c) Calculated Raman spectra for $325 \mathrm{~nm}$ light excitation and identical carrier concentrations as in (b). 


\section{Raman spectrum of Cs doped graphene/Ir(111)}

We have used a Fano lineshape ${ }^{30,36,58,59}$ to fit the $G$ band Raman spectra for all doping levels by

$$
F(\omega)=I_{0} \frac{\left(1+\frac{\omega-\omega_{0}}{q \gamma / 2}\right)^{2}}{1+\left(\frac{\omega-\omega_{0}}{\gamma / 2}\right)^{2}} .
$$

Here $I_{0}$ is the Raman intensity, $\omega_{0}$ the line position, $\gamma$ the full width at half maximum and $1 / q$ the asymmetry (or Fano) factor which describes the strength of the interference effect between the discrete and continuous spectra. For $1 / q=0$, we have a Lorentzian lineshape indicating no interference effect. In the following we analyze the carrier concentration dependence of $I_{0}, \omega_{0}, \gamma$ and $1 / q$. Figure 3a illustrates that, upon evaporation of Cs onto the sample, the observed Raman spectra dramatically change compared to those of pristine graphene. For the first deposition of Cs all laser energies except the red laser (633 $\mathrm{nm})$ yield a finite Raman intensity. We attribute the appearance of a Raman signal to removal of the hybridization of the graphene and Ir states as discussed in the ARPES section. From Figure 3a we observe that the Raman intensity almost vanishes for the green (532 nm) laser at a carrier concentration of $2.4 \times 10^{14} \mathrm{~cm}^{-2}$ (corresponding to the $\sqrt{3} \times \sqrt{3}$ phase). The Raman intensity for the blue laser $(442 \mathrm{~nm})$ vanishes at a doping level of $3.1 \times 10^{14} \mathrm{~cm}^{-2}$. On the other hand, the UV laser $(325 \mathrm{~nm})$ yields a Raman spectrum up to the highest doping level. This can be understood in terms of the condition that light can only induce transitions across the Dirac cone between occupied states in the valence band and unoccupied states in the conduction band. If doping shifts the Fermi level deep into the conduction band, these transitions are forbidden by Pauli blocking. The UV laser always fulfills the resonance condition $2 E_{F}>E_{l a s e r}$ since its laser energy $\left(E_{l a s e r}=3.8 \mathrm{eV}\right)$ is significantly higher than twice the maximum Fermi level shift $\left(E_{F}=1.58 \mathrm{eV}\right.$ from the ARPES data of maximally doped graphene).

Figure $3 a$ also reveals that the Raman spectrum taken with the lowest photon energy for each doping level becomes Fano-like. This applies to the $532 \mathrm{~nm}$ (green) laser for $1.5 \times 10^{14} \mathrm{~cm}^{-2}$, the $442 \mathrm{~nm}$ (blue) laser for $2.4 \times 10^{14} \mathrm{~cm}^{-2}$ and the UV laser for $3.1 \times 10^{14} \mathrm{~cm}^{-2}$. The most 
striking feature is that the Fano tail of the present data is towards higher wavenumber with respect to the peak position. This corresponds to a positive sign of $1 / q$. The origin of this unusual Fano lineshape will be explained in the next section. In Figure 3b we show UV Raman spectra with increasing carrier concentration. The position of the $G$ peak shifts towards higher phonon energies for carrier concentrations up to $1.5 \times 10^{14} \mathrm{~cm}^{-2}$ before it shifts down. The UV Raman spectrum allows for comparison of the $G$ line position of pristine (1606.3 $\left.\mathrm{cm}^{-1}\right)$, weakly doped graphene $\left(1615.9 \mathrm{~cm}^{-1}\right.$ for the lowest Cs deposition) and fully doped graphene $\left(1550.0 \mathrm{~cm}^{-1}\right)$. A key to understanding the present results is the interference of the electronic and vibrational Raman which also plays a major role in explaining the Fano asymmetry in carbon nanotubes. ${ }^{28}$ Here we apply this theory ${ }^{30}$, using the experimental band structure of graphene derived from ARPES measurements. The calculated Raman spectra are depicted in Figure $3 \mathrm{c}$ and a very good agreement regarding the position and Fano asymmetry can be seen. In the following section we will show a quantitative comparison between experiment and theory regarding the position and asymmetry of the $G$ band and discuss the details of the Raman calculation. Let us now look to the temperature dependent Raman spectra of doped graphene. This is motivated by question if intercalation of Cs liberates graphene from the substrate, so that it does not follow any more the lattice constant of Ir. The corresponding Raman spectra and the $G$ band positions are shown in Figure 4. An upshift of the $G$ band position by $7 \mathrm{~cm}^{-1}$ with decreasing temperature (from $300 \mathrm{~K}$ to $5 \mathrm{~K}$ ) is found. This is within the experimental accuracy to what we observed in Figure 2 for pristine graphene/Ir. Thus, despite Cs is intercalated in between graphene and $\operatorname{Ir}(111)$, the graphene still follows the compression of the underlying Ir substrate.

\section{Discussion}

\section{Electronic Raman scattering}

In the electronic Raman scattering, the photoexcited electron and hole couple to electronic 

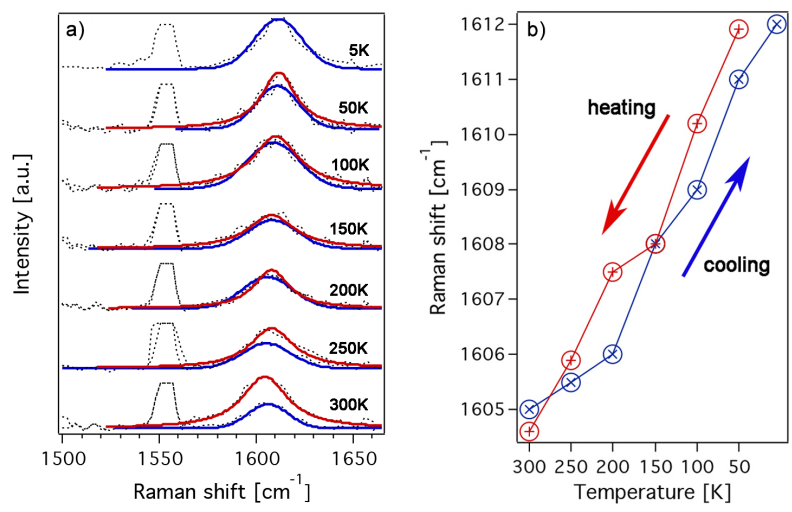

Figure 4: (a) Temperature dependent Raman spectra of the $\sqrt{3} \times \sqrt{3}$ phase of Cs doped graphene for a cooling and a warming-up cycle. (b) Raman peak positions as a function of temperature.

excitations via the Coulomb interaction and can generate one or more electron-hole pairs. The large density of states in heavily doped graphene around the $M$ point enhances the cross section for electronic Raman scattering. In the Figure 5(a,b) we graphically depict the relaxation processes which we consider in the calculation. We consider first order (creation of one electron-hole pair) and second order (creation of two electron-hole pairs) processes. The Coulomb interaction is affected by the dielectric screening of the substrate which is strong for the Ir substrate $(\epsilon=50)^{60}$. As a consequence, only the first-order process (wavevector $\mathbf{q}=0$ ) is dominant for Ir. This is depicted in Figure 5c. We note that this first-order process excites an intraband electron-hole pair whose Coulomb interaction is maximum at wavevector $\mathbf{q}=0$. This process is completely different from the interband electron-hole pair excitation in the low doping regime, in which the direct Coulomb interaction vanishes at $\mathbf{q}=0^{31}$.

Interference between the first-order electronic Raman scattering (ERS) (shown by the green line) and the $\mathrm{G}$ band (red line) produces the asymmetric Fano lineshape towards larger wavenumber $(1 / q>0)$. Figure 5 d depicts the simulated Raman spectra of highly doped graphene on $\mathrm{SiO}_{2}$ substrate. Due to the relatively weak screening effect $(\epsilon=4)$, the second order Raman $(\mathbf{q} \neq 0)$ process overcomes the first-order process thanks to the double resonant effect. The resulting Raman spectra are asymmetric towards the lower wavenumber 
$(1 / q<0)$. The spectra reported for doped graphene on Si have the Fano tail towards low wavenumers ${ }^{27,36,37}$ corresponding to negative values of $1 / q$. Our calculated results can thus fully explain the present data on Ir and the literature data on silicon wafers and other insulators.
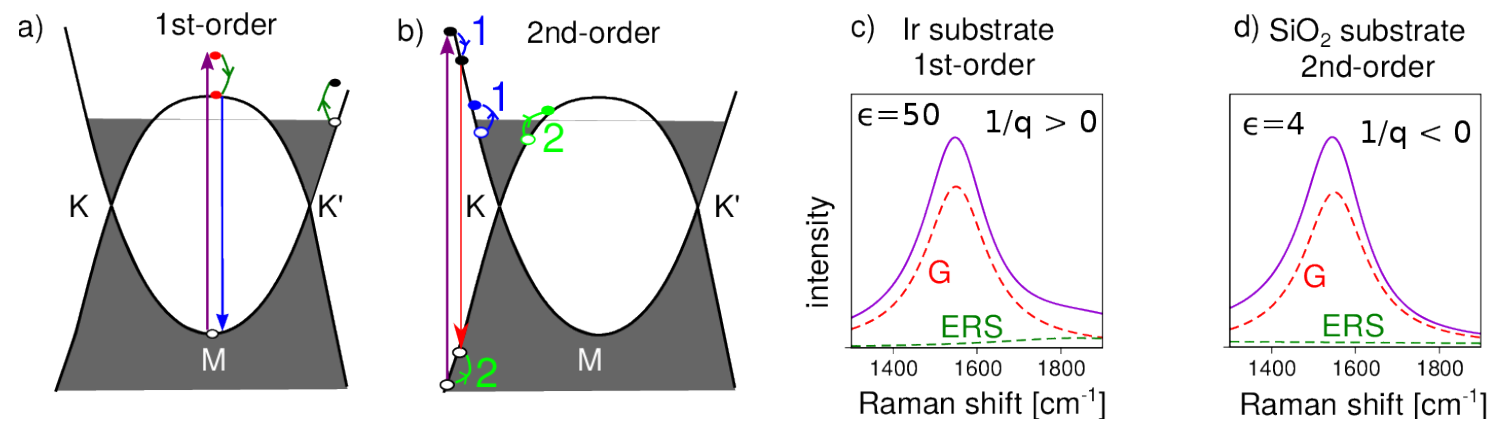

Figure 5: (a) In the first-order electronic Raman scattering (ERS) process, an electron is excited to a virtual state above the saddle-point energy and then relaxes via Coulomb interaction by exciting an e-h pair. When the electron recombines with the hole, the scattered energy is resonant to the $M$ point energy. (b) In the second-order ERS process the photoexcited e-h pair occupies a real state. The electron and hole relax to lower energy states by exciting two e-h pairs near Fermi surface with oppposite momenta. Calculated Raman spectra of highly doped-graphene on (c) Ir substrate and (d) $\mathrm{SiO}_{2}$ substrates. The dashed lines indicate the spectral contributions of the vibrational Raman scattering by the $G$ phonon and ERS. The solid line is the spectrum after considering interference between these two contributions.

\section{$\mathrm{G}$ band position: experiment and theory}

In Figure 6a, we show plots of the experimental $G$ band positions versus carrier concentration and along with a calculation of the phonon energy shift with carrier concentration. We quantitatively describe the observed Raman $G$ band shifts by considering the effects of phonon renormalization and lattice expansion ${ }^{5}$. Our model is based on expressing the doping induced change of the Raman $G$ band frequency $\Delta \omega$ as a sum of static (lattice expansion) and dynamic (electron-phonon coupling) effects as $\Delta \omega=\alpha \omega_{\text {static }}+\omega_{\text {dynamic }}\left(D^{2}\right)$ (Ref. 5). Here $\alpha$ is a parameter that describes the scaling of the theoretical doping dependence for freestanding graphene due to the effects of the Ir substrate. We have shown in Figure 2 (temperature dependence) that the lattice constant of graphene perfectly follows the Ir substrate. Due to the strong interaction, graphene's expansion due to doping will also be 
affected and hence $\alpha \neq 1 . D^{2}$ is the deformation potential as explained in the ARPES section. The equations for $\omega_{\text {static }}$ and $\omega_{\text {dynamic }}$ are given in the Methods sections. Using the ARPES-derived value of $D^{2}=63.1 \mathrm{eV}^{2} / \AA^{2}$, we proceed to perform the fit of the parameter $\alpha$ describing the lattice expansion versus carrier concentration. We find $\alpha=0.18$ which would suggest there is still compressive strain, i.e. the lattice constant of freestanding graphene doped to an equal concentration would be larger. Let us discuss these results for $D^{2}$ and $\alpha$ in more detail. First, the experimental value of $D^{2}$ is considerably larger than the DFT value of $D^{2}=45.6 \mathrm{eV}^{2} / \AA^{2}$ (Ref. 5). A perfect agreement between theory and experiment is achieved by $G W$ calculations which yield $D^{2}=62.8 \mathrm{eV}^{2} / \AA^{2}$ (Ref. 61). This can be understood by the fact that the underlying electron and phonon dispersions of graphene are accuratly described only by $G W$ calculations and DFT underestimates the size of the electron and phonon dispersions. ${ }^{61}$ Regarding the dependence of the $\alpha \omega_{\text {static }}$ term that describes the lattice expansion on carrier concentration, we estimate from Figure 6a that the observed maximum $G$ band frequency downshift from undoped graphene is $\sim 50 \mathrm{~cm}^{-1}$. Assuming that $69 \mathrm{~cm}^{-1}$ downshift of the $G$ band phonon corresponds to $1 \%$ expansive strain (Ref. 57 ), we estimate a C-C bond increase of $0.8 \%$. Indeed, this is close to what has been observed by diffraction in intercalated graphite where the C-C bond length increases from $1.4211 \AA$ (pristine graphite) to $1.4320 \AA$ (stage 1 GIC) ${ }^{62}$ corresponding to a $0.7 \%$ increase in the lattice constant. The small differences to the present case could be ascribed to the higher doping level that we have achieved in the present case corresponding to a shift of the charge neutrality point to $E_{D}=1.58 \mathrm{eV}$ whereas the stage $1 \mathrm{KC}_{8}$ GIC has $E_{D}=1.35 \mathrm{eV} \cdot{ }^{63}$. Interestingly, the theoretically expected downshift for the carrier concentrations achieved is much larger which is described by $\alpha=0.18$. We attribute this to two effects. First, the substrate interaction is strong despite Cs is intercalated in between graphene and the substrate. This is evident from the temperature dependent Raman spectra of the $\sqrt{3} \times \sqrt{3}$ phase where the C-C lattice constant is following the Ir substrate. It is clear that the substrate interaction can hinder the lattice expansion which would result in $\alpha<1$, as we have observed. Additionally substrate 
interaction can cause the formation of wrinkles. Second, regarding the very large difference between experiment and theory, the analytical expression for $\omega_{\text {static }}$ is derived from DFT calculations and perhaps not sufficiently accurate to describe the very high doping levels considered here. ${ }^{5}$

Next, let us discuss how our observed $G$ band shift corresponds to experiments performed with ionic liquid gated graphene (data from Ref. 15). These data are shown in Figure 6a along with our experiments. The ionic liquid gated graphene on $\mathrm{Si}$ oxide has a maximum upshift of $25 \mathrm{~cm}^{-1}$ (Ref. 15) whereas the observed maximum $G$ band upshift of Cs doped graphene on Ir is about $10 \mathrm{~cm}^{-1}$. Notably, Rb doped graphene on $\mathrm{Si}$ oxide also displayed a maximum upshift of the $G$ band by $25 \mathrm{~cm}^{-1}$ (Ref. 37 ) and it could therefore be related to the substrate. We believe that the observed differences in the slope of the $G$ band with doping lie in the phonon dispersion relation of graphene on insulators and on metals. It has been put forward, that for graphene on metals, the Kohn anomalies at $\Gamma$ and $K$ points are screened by the metal substrate ${ }^{64}$. High resolution electron energy loss spectroscopy measurements and calculations of the phonon dispersion relations of the graphene/ $\operatorname{Ir}(111)$ system ${ }^{65}$ indicate that the Kohn anomaly at $\Gamma$ is not as strongly kinked than in freestading graphene. This results in a higher phonon frequency for graphene/ $\operatorname{Ir}(111)$ compared to freestanding graphene. Upon doping, the Kohn anomaly at $\Gamma$ point shifts away to a finite wavevector equal to $2 k_{F}$ ( $k_{F}$ is length of the Fermi wavevector) and the $\Gamma$ point phonon frequency moves to higher values. Thus, for graphene on Ir, the upshift in phonon energy as a result of doping is smaller than the one observed for freestanding graphene. These arguments explain the experimental data of graphene/Ir(111) and graphene/Si oxide shown in Figure 6a.

\section{Doping dependence of Fano asymmetry and spectral linewidth}

Figure $6 \mathrm{~b}$ depicts the experimental and theoretical Fano asymmetry parameter $1 / q$ and the experimental spectral width (FWHM) as a function of carrier concentration. The values of Fano asymmetry $1 / q$ and the FWHM at carrier concentrations larger than $3 \times 10^{14} \mathrm{~cm}^{-2}$ 
exhibit a strong deviation from the behaviour at lower carrier concentrations. Correlating this with the experimental Fermi surfaces from ARPES, we attribute the sudden increase in $1 / q$ and line width to increased electronic Raman scattering as the Lifshitz transition is approached. This is not only via the large carrier concentration which causes the downshift of the $G$ band position but also via the change in the energy-momentum conservation for electron-hole pair excitation affecting the Fano lineshape of the $G$ band as theoretically predicted $^{30}$.

(a) Cs - graphene/lr phase
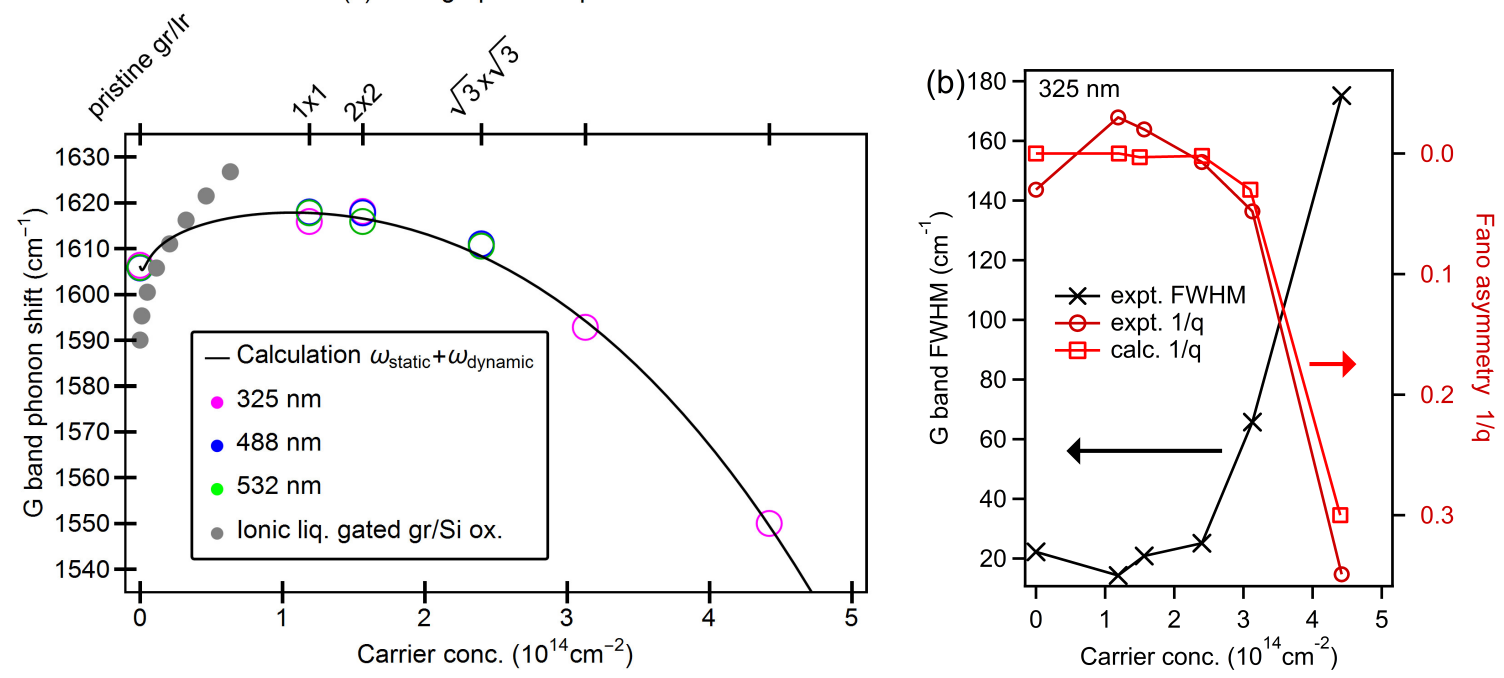

Figure 6: (a) Raman $G$ peak positions of Cs doped graphene versus the carrier concentration. The full line is a model calculation of the $G$ band frequency (see Methods section) and open circles are the experimentally determined $G$ band maxima. The grey filled circles indicate the $G$ peak position from ionic liquid gated graphene on fused silica (Ref. 15). (b) Crosses connected by a black line: expt. linewidth (full width at half maximum) of the Raman $G$ peak. Circles connected by a dark red line: the experimental Fano asymmetry factor obtained from measurements with the UV $(\lambda=325 \mathrm{~nm})$ laser. The squares connected by a red line are the calculated values of the Fano asymmetry (obtained by fitting a Fano line to the calculated spectra in Figure 3c).

\section{Conclusions and outlook}

In conclusion, we have established a fully experimental relation between energy shift and Fano asymmetry parameter of the Raman $G$ band versus carrier concentration in doped epi- 
taxial graphene. This is based on ARPES experiments that reveal the deformation potential $D^{2}$ from the kink feature and Raman measurements on identical samples. This relation is expected to be useful for future stand-alone UHV Raman experiments. In the Raman experiments we have exploited the band renormalization upon doping which reduces the optical transition energy at the saddle point. This allowed us to achieve resonance Raman conditions with UV light in the vicinity of the van Hove singularity. We have observed a peculiar Fano lineshape with an asymmetry tail towards high Raman shifts. This is opposite to what is known for doped graphene on semiconductors such as Si oxide. By performing resonant Raman calculations, we have fully explained this behaviour by considering first and second order electronic Raman contributions.

Our work has introduced UV UHV Raman spectroscopy as a function of temperature as a versatile tool for surface science of two-dimensional materials. Let us now consider two future research directions. First, the presented approach could also be applied to hole doped graphene. Theoretically, we expect it to yield qualitatively similar results if the Fermi level reaches the van-Hove singularity in the valence band. Hole doping of graphene on $\operatorname{Ir}(111)$ has already been achieved by oxygen intercalation ${ }^{66}$ or chlorine intercalation ${ }^{67}$ and it would be interesting to investigate such samples by UHV Raman spectroscopy at low temperatures. Second, UHV Raman spectroscopy of alkali doped graphene could be used for investigation of the superconducting properties of graphene analogous to previous experiments that are carried out on superconducting bulk $\mathrm{CaC}_{6}$ (Ref. 68). In these experiments, a sharp superconducting coherence peak is observed by Raman at $24 \mathrm{~cm}^{-1}$ which is very close to the value for the superconducting gap obtained by scanning tunneling spectroscopy $\left(25.8 \mathrm{~cm}^{-1}\right)$ highlighting that this Raman peak has its origin in the superconducting phase. ${ }^{68}$ For graphene, electronic Raman scattering from the superconducting phase has not been observed yet. It should also lead to low-energy Raman peaks with an energy roughly equal to the size of the superconducting gap. ${ }^{69}$ Theory predicts that this holds for both, $s$-wave and $d$-wave superconducting graphene at doping levels close to what is shown in the present work. ${ }^{70}$ 
The Raman intensity of these low-energy peaks has been estimated to be approximately a factor 1000 lower than the $G$ band intensity. ${ }^{69}$ Given, the comparably low Raman intensity of graphene on metals with respect to graphene on insulators, we estimate that the electronic Raman peaks at low wavenumber will be hard to measure for the present graphene/Ir(111) system. An approach for addressing this problem is to carry out the present experiment with graphene transferred onto an an insulating substrate. The large Raman response of graphene on insulators compared to graphene on metals could be a key to measure the appearance of low wavenumber Raman peaks when the temperature is below $T_{c}$. Thus, a future experiment could be to perform doping graphene into the superconducting state after it is transferred onto an insulator. Having doped graphene on an insulator inside UHV would in principle also allow for electrical transport characterization. To that end, the presented UHV Raman setup can be extended via electrical feedthroughs into the UHV chamber. In such a setup, the Raman spectrum and the four-point resistance could be measured simultaneously as a function of alkali doping. This could provide strong evidence for the existence of of a superconducting phase and elucidate its Raman response. Finally, such a work could also be extended to doped bilayer graphene with a relatively higher critical temperature ${ }^{21}$ and doped heterostructures composed out of different van-der-Waals materials.

\section{Methods}

\section{Synthesis}

Graphene/Ir(111) has been synthesized in-situ in the preparation chamber attached to the UHV Raman system, using an established recipe ${ }^{71}$ that yields monolayer coverage by a selflimiting process. ${ }^{72}$ The fact that we have monolayer graphene is supported by three techniques: ARPES, STM and LEED. ARPES spectra show only one $\pi$ valence band. A bilayer, e.g. would show two $\pi$ valence bands. We have also verified that the full $\operatorname{Ir}(111)$ is covered by monolayer graphene via scanning the spot of the ARPES measurement (spotsize: 100×50 
$\mu m^{2}$ ) over the full $1 \times 1 \mathrm{~cm}^{2} \operatorname{Ir}(111)$ crystal. We have nowhere found two valence bands which would hint bilayer formation. The STM images that we took of samples prepared in that way (supporting information Figure S4) show the moiré pattern due to substrate interaction. This moiré pattern is a clear proof of monolayer coverage. Also, the individual carbon atoms of the monolayer are clearly seen. Finally, the LEED shown in Figure S2 of the supporting information displays the diffraction spots due to the moiré pattern of monolayer graphene/Ir(111). The $\operatorname{Ir}(111)$ single crystal which was used as a substrate for the graphene synthesis which was first sputtered $(1 \mathrm{keV})$ in Ar atmosphere $\left(1 \times 10^{-6} \mathrm{mbar}\right)$ followed by an annealing step under $\mathrm{O}_{2}$ flow $\left(1 \times 10^{-7} \mathrm{mbar}\right)$ at $1200^{\circ} \mathrm{C}$ for $30 \mathrm{mins}$. When the crystal cooled down to room temperature a rapid flashing to $1700^{\circ} \mathrm{C}$ provided a clean surface indicated by a sharp hexagonal LEED pattern. For the graphene synthesis we used a combination of CVD (chemical vapor deposition) and TPG (temperature programmed growth). Hereby propene $\left(\mathrm{C}_{3} \mathrm{H}_{6}\right)$ was dosed into the UHV chamber $\left(1 \times 10^{-6} \mathrm{mbar}\right)$ for $60 \mathrm{~s}$ at room temperature to adsorb molecules on the iridium surface followed by a flashing step to $1250^{\circ} \mathrm{C}$ for $3 \mathrm{~min}$ without propene to create graphene islands with the same crystallographic orientation as the substrate. The TPG synthesis was applied twice to increase the amount of graphene islands. After the second TPG step the sample was not cooled down to room temperature but only to $900^{\circ} \mathrm{C}$. After reaching this temperature, the CVD growth was carried out. Hereby propene was dosed $\left(1 \times 10^{-6} \mathrm{mbar}\right)$ for $15 \mathrm{~min}$ to grow graphene in the areas between the islands creating a closed monolayer of graphene without rotational domains with respect to iridium. Finally the sample was cooled down slowly to room temperature to minimize the formation of wrinkles. Cs was deposited by evaporation from a commercial SAES getter source. The evaporated amount of Cs was calibrated by a quartz crystal microbalance. The amount of evaporated Cs monolayer reported in the paper are with respect to the bulk Cs structure. 


\section{Angle-resolved photoemission spectroscopy}

ARPES was performed at the BaDElPh beamline ${ }^{73}$ of the Elettra synchrotron in Trieste (Italy) with linear s- and p- polarisation at $h \nu=31 \mathrm{eV}$ at temperatures of $20 \mathrm{~K}$. The graphene/Ir(111) samples were prepared in-situ and measured in a vacuum better than $5 \times 10^{-11}$ mbar. Immediately after the synthesis, Cs deposition was carried out in an ultra-high vacuum (UHV) chamber from SAES getters with the sample at RT. We performed stepwise evaporation of Cs which we monitored by ARPES measurements of the band structure. Cs evaporation was stopped after the desired doping level was reached.

\section{Scanning Tunneling Spectroscopy and Microscopy}

STM and STS are carried out with a background pressure lower than $10^{-11}$ mbar. The constant energy maps are recorded using the lock-in technique with a modulation frequency of $833.1 \mathrm{~Hz}$ and a modulation amplitude of $8 \mathrm{mV}$, providing an energy resolution of $14 \mathrm{meV}$. An etched tungsten tip is used for all measurements, which is prepared in situ by applying positive or negative voltage pulses up to $10 \mathrm{~V}$. Fourier transformed images are obtained from spectroscopic maps by using the fast Fourier transform of the SPIP software ${ }^{74}$ with Hanning window. Subsequently, the symmetry of the sample is exploited to enhance the signal to noise ratio.

\section{Ultra-high Vacuum Raman Spectroscopy}

UHV Raman measurements were performed in the back-scattering geometry using commercial Raman systems (Renishaw) integrated in a homebuilt optical chamber ${ }^{75}$, where the exciting and Raman scattered light were coupled into the vacuum using a 50x long-working distance microscope objective with an NA of $\sim 0.4$ and a focal distance of $20.5 \mathrm{~mm}$ for lasers with wavelength $442 \mathrm{~nm}, 532 \mathrm{~nm}$ and $633 \mathrm{~nm}$. For the UV laser, UV compatible optical elements have been used. The $20 x \mathrm{UV}$ objective has a focal distance equal to $13 \mathrm{~mm}$ and 
an $\mathrm{NA}=0.32$. A sketch of our experimental setup is shown in the supplemental information. The laser powers used were $\sim 2 \mathrm{~mW}$ for the UV laser and $9 \mathrm{~mW}, 25 \mathrm{~mW}$ and $45 \mathrm{~mW}$ for blue, red, and green lasers respectively. Assuming that this energy gets spread over $\sim 4 \mu \mathrm{m}^{2}$, we obtain power densities of $100 \mathrm{~kW} / \mathrm{cm}^{2}$. Using lHe cooling and given the fact that graphene is directly on a metal, these laser powers result in a linear dependence of Raman intensity to the laser power. The position of the laser on the sample could be checked by a camera in the laser path. All spectra have been calibrated in position and intensity to the $\mathrm{O}_{2}$ vibration at $1555 \mathrm{~cm}^{-1}$ (Ref. 76). $\mathrm{O}_{2}$ Raman peaks can be seen with all laser lines used in the present experiment which is consistent with the previous published works. ${ }^{76,77}$ Further precautions that we took in order to prevent laser heating induced effects is a study of the laser power dependence (see supporting information).

\section{Calculations of the Raman spectra}

\section{Calculations of the doping dependent phonon shift}

We have calculated the Raman shift $\Delta \omega=\alpha \omega_{\text {static }}+\omega_{\text {dynamic }}\left(D^{2}\right)$ according to the wellestablished model ${ }^{5}$. The frequency downshift due to doping induced lattice expansion is described by $\omega_{\text {static }}$. The parameter $\alpha$ is a scaling factor for the phonon downshift with respect to the calculated doping dependence of freestanding graphene. The frequency upshift is described by $\omega_{\text {dynamic }}\left(D^{2}\right)$. Here $D^{2}$ is the deformation potential. For $\omega_{\text {static }}$ we use the equation for freestanding graphene (Ref. 5):

$$
\omega_{\text {static }}=-2.13 \sigma-0.0360 \sigma^{2}-0.00329 \sigma^{3}-0.226|\sigma|^{3 / 2}
$$

Here $\sigma$ is the charge carrier density per $\mathrm{cm}^{2}$ and $\omega_{\text {static }}$ is given in $\mathrm{cm}^{-1}$. For calculation of the dynamic contribution, we need to consider

$$
\widetilde{F}_{\mathbf{q}}^{\epsilon_{F}}(\omega)=\frac{2}{N} \sum_{\mathbf{k}, n \neq m} \frac{D^{2}\left(\tilde{f}_{\mathbf{k} m}-\tilde{f}_{\mathbf{k} n}\right)}{\epsilon_{\mathbf{k}, m}-\epsilon_{\mathbf{k}, n}+\hbar \omega+i \delta}
$$


Here the sum goes over all points in the 2D BZ and $\widetilde{f}_{\mathbf{k} m}=f\left(\epsilon_{\mathbf{k} m}-\epsilon_{F}\right)$ with $f$ being the Fermi distribution function. For numerical integration, we have used a trigonal grid having $\sim 1000$ points in the $2 \mathrm{D} \mathrm{BZ}$ of graphene. $\hbar \omega$ is the phonon energy of the undoped system and $\delta=10 \mathrm{meV}$ a small broadening term. For the band structure calculations we have used a third nearest neighor tight-binding fit to the experimental ARPES band structure. ${ }^{46}$ The corresponding dynamic shift is calculated by

$$
\omega_{\text {dynamic }}=\operatorname{Re}\left[\frac{\widetilde{F}_{\mathbf{0}}^{\epsilon_{F}}\left(\omega_{0}\right)-\widetilde{F}_{\mathbf{0}}^{0}\left(\omega_{0}\right)}{2 M \omega_{0}}\right] .
$$

Here $M$ is the free electron mass, $\omega_{0}$ the unperturbed phonon frequency of the $G$ band and

$\widetilde{F}_{\mathbf{0}}^{\epsilon_{F}}$ is defined in equation 3 . In the calculations we used an artificially high temperature of $T=400 \mathrm{~K}$ in the Fermi distribution function as a means to describe doping inhomogeneities and charge puddles that can not be resolved spatially. A similar observation was made in previous works that have also used artificially high temperatures ${ }^{37}$ or Fermi level smearing ${ }^{16}$.

\section{Raman Intensity calculation}

We consider the interference between the $G$ band phonon Raman and electronic Raman scattering (ERS) pathways and write the Raman intensity as

$$
I\left(\omega_{\mathrm{s}}\right)=\left[A_{G}\left(\omega_{\mathrm{s}}\right)+A_{\mathrm{ERS}}\left(\omega_{\mathrm{s}}\right)\right]^{2}
$$

where $A_{G}=\sum_{\nu} A_{\nu}$, is the $G$ phonon scattering amplitude which consists of zone center $(\Gamma$ point) $\nu=\mathrm{LO}$ and iTO modes and $A_{\mathrm{ERS}}$ is the ERS scattering amplitude. The phonon Raman process consists of (1) excitation of an electron-hole pair by the electron-photon interaction, (2) phonon emission by means of the electron-phonon interaction, and (3) electron-hole recombination and photoemission by the electron-photon interaction. Based on the three 
sub-processes, phonon scattering amplitude is given by ${ }^{78}$

$$
A_{\nu}\left(E_{\mathrm{s}}\right)=\sum_{\mathbf{k}} \frac{M_{\mathrm{op}}^{\mathrm{vc}}(\mathbf{k}) M_{\mathrm{ep}}^{\nu}(\mathbf{k}, \mathbf{k}) M_{\mathrm{op}}^{\mathrm{cv}}(\mathbf{k})\left[f\left(E_{\mathbf{k}}^{\mathrm{v}}\right)-f\left(E_{\mathbf{k}}^{\mathrm{c}}\right)\right]}{\left[E_{\mathrm{L}}-E_{\mathbf{k}}^{\mathrm{cv}}-i \gamma / 2\right]\left[E_{\mathrm{L}}-E_{\mathbf{k}}^{\mathrm{cv}}-\hbar \omega^{\nu}-i\left(\gamma+\Gamma_{\nu}\right) / 2\right]\left[E_{\mathrm{L}}-\hbar \omega^{\nu}-E_{\mathrm{s}}-i \Gamma_{\nu} / 2\right]}
$$

Here $E_{\mathrm{L}}$ is the laser energy, $E_{\mathrm{s}}$ is the scattered photon energy, $E_{\mathbf{k}}^{\mathrm{cv}}=E_{\mathbf{k}}^{\mathrm{c}}-E_{\mathbf{k}}^{\mathrm{v}}$ is the electron energy difference between the conduction (c) and the valence (v) bands at a wave vector k. The energy bands of graphene have been obtained by the tight-binding (TB) fits to the experimental band structure considering up to the three nearest-neighbors for each of doping level. The $M_{\mathrm{op}}$ and $M_{e p}^{\nu}$ are the electron-photon and electron-phonon matrix elements, respectively. These matrix elements are obtained within the TB method. ${ }^{79,80}$. The phonon frequency of the $\nu$-th mode is depicted by $\omega^{\nu}$ and a broadening factor of the photoexcited carriers $\gamma=0.2 \mathrm{eV}$ is used. The phonon linewidth $\Gamma_{\nu}$ is fitted to the Raman measurements. The summation of states considered in Eq. (6) are taken below a cut-off energy $E_{\mathbf{k}}^{\mathrm{cv}}=5 \mathrm{eV}$. For the ERS amplitude $A_{\mathrm{ERS}}$, we consider the lowest order processes as shown in Figure 4. In the first-order ERS, the photo-excited carrier excites an electron-hole (e-h) pair via Coulomb interaction with zero momentum transfer $(\mathbf{q}=0)$ or vertical transition. We note that the e-h pair is allowed to occupy a virtual state as the lifetime of Coulomb interaction is very short $(\sim 10 \mathrm{fs})$. The first order ERS amplitude is given by

$$
A_{\mathrm{ERS}}^{(1)}\left(E_{\mathrm{s}}\right)=\sum_{\mathbf{k}} \sum_{\mathbf{k}^{\prime}} \frac{M_{\mathrm{op}}^{\mathrm{vc}}(\mathbf{k}) K_{\mathbf{k c}, \mathbf{k}^{\prime} \mathbf{c}, \mathbf{k c}, \mathbf{k}^{\prime} \mathrm{c}}(0) M_{\mathrm{op}}^{\mathrm{cv}}(\mathbf{k})\left[f\left(E_{\mathbf{k}}^{\mathrm{v}}\right)-f\left(E_{\mathbf{k}}^{\mathrm{c}}\right)\right]}{\left[E_{\mathrm{L}}-E_{\mathbf{k}}^{\mathrm{cv}}-i \gamma / 2\right]\left[E_{\mathrm{L}}-E_{\mathbf{k}}^{\mathrm{cv}}-E_{\mathbf{k}^{\prime}}^{\mathrm{e}}-i\left(\gamma+\Gamma_{\mathrm{e}}\right) / 2\right]\left[E_{\mathrm{L}}-E_{\mathbf{k}^{\prime}}^{\mathrm{e}}-E_{\mathrm{s}}-i \Gamma_{\mathrm{e}} / 2\right]},
$$

where $E_{\mathbf{k}^{\prime}}^{\mathrm{e}}$ and $\Gamma_{\mathrm{e}}=60 \mathrm{meV}$ are the energy of the excited e-h pair and the Coulomb scattering rate, respectively. If $E_{\mathrm{L}}>E_{M}$ (here $E_{M}$ is the $M$ point transition energy), we expect that the photoexcited electron relaxes to the conduction band at the $M$ point. In such a way we estimate $E_{\mathrm{s}}=E_{M}-E_{e x}$, where and $E_{e x}$ is the exciton binding energy, estimated to be $100 \mathrm{meV}$. For $E_{\mathrm{L}}<E_{M}$, we expect hot luminescence ${ }^{15}$, i.e. the photo-excited carrier relaxes to the lowest intermediate energy $\sim 2 E_{F}$ and then recombines with the hole by emitting 
$E_{s} \approx 2 E_{F}$. The direct Coulomb interactions between two electrons for initial states 1,2 with states 3,4 is given by $K_{1,2,3,4}(\mathbf{q})$. In the TB approximation this kernel is expressed as

$$
K_{1,2,3,4}(\mathbf{q})=\sum_{s s^{\prime}=\mathrm{A}, \mathrm{B}} C_{s}^{1} C_{s^{\prime}}^{2} C_{s}^{* 3} C_{s^{\prime}}^{* 4} v(\mathbf{q}) / \epsilon
$$

Here $C_{s}^{i}$ is the TB coefficient for the atomic site $s$ at the state $i$ and $v(\mathbf{q})$ is the Fourier transform of the Ohno potential ${ }^{81}$. $\epsilon$ is the dielectric constant of the substrate. Because the Raman shift of the ERS process is about $0.2 \mathrm{eV}$, we do not consider the dynamical screening effect. For the second order ERS, we consider the excitation of two e-h pairs by the photo-excited electron and hole [Figure 4(b)]. The amplitude of the second-order ERS process is given by

$$
\begin{aligned}
A_{\mathrm{ERS}}^{(2)}\left(E_{\mathrm{s}}\right)= & \sum_{\mathbf{k}} \sum_{\mathbf{k}^{\prime} \mathbf{k}^{\prime \prime} \mathbf{q}} \frac{M_{\mathrm{op}}^{\mathrm{vc}}(\mathbf{k}) K_{(\mathbf{k}+\mathbf{q}) \mathrm{v}, \mathbf{k}^{\prime \prime} \mathrm{c}, \mathbf{k v}, \mathbf{k}^{\prime \prime}+\mathbf{q c}}(-\mathbf{q})}{\left[E_{\mathrm{L}}-E_{\mathbf{k}}^{\mathrm{cv}}-i \gamma / 2\right]\left[E_{\mathrm{L}}-E_{\mathbf{k}}^{\mathrm{cv}}-E_{\mathbf{k}^{\prime}}^{1}-E_{\mathbf{k}^{\prime \prime}}^{2}-i\left(\gamma+2 \Gamma_{\mathrm{e}}\right) / 2\right]} \\
& \times \frac{K_{\mathbf{k c}, \mathbf{k}^{\prime} \mathbf{c},(\mathbf{k}+\mathbf{q}) \mathrm{c},\left(\mathbf{k}^{\prime}-\mathbf{q}\right) \mathrm{c}}(\mathbf{q}) M_{\mathrm{op}}^{\mathrm{cv}}(\mathbf{k})\left[f\left(E_{\mathbf{k}}^{\mathrm{v}}\right)-f\left(E_{\mathbf{k}}^{\mathrm{c}}\right)\right]}{\left[E_{\mathrm{L}}-E_{\mathbf{k}^{\prime}}^{1}-E_{\mathrm{s}}-i \Gamma_{\mathrm{e}} / 2\right]\left[E_{\mathrm{L}}-E_{\mathbf{k}^{\prime}}^{1}-E_{\mathbf{k}^{\prime \prime}}^{2}-E_{\mathrm{s}}-i 2 \Gamma_{\mathrm{e}} / 2\right]} .
\end{aligned}
$$

Here $E_{\mathbf{k}^{\prime}}^{1}=E_{\mathbf{k}^{\prime}-\mathbf{q}}^{\mathrm{c}}-E_{\mathbf{k}^{\prime}}^{\mathrm{c}}$ and $E_{\mathbf{k}^{\prime \prime}}^{2}=E_{\mathbf{k}^{\prime \prime}+\mathbf{q}}^{\mathrm{c}}-E_{\mathbf{k}^{\prime \prime}}^{\mathrm{c}}$ are the excitation energies of the e-h pairs. 


\section{Acknowledgements}

M.H., N.E., B.V.S. and A.G. acknowledge the ERC grant no. 648589 'SUPER-2D', funding from DFG projects A01 with CRC 1238 and GR 3708/2-1. A.G. ackowledges INST 216/8081 FUGG, support from the "Quantum Matter and Materials" (QM2) initiative and support from Tohoku University for a research stay in Japan. The research leading to these results has received funding from the Horizon 2020 EC programme under grant agreement n. 730872 (CALIPSOplus). This work has been supported by the University of Cologne through the Institutional Strategy of the University of Cologne within the German Excellence Initiative. R.S. acknowledges MEXT KAKENHI (Nos. JPI8H01810, JP15K21722).

\section{References}

(1) Ferrari, A. C.; Meyer, J. C.; Scardaci, V.; Casiraghi, C.; Lazzeri, M.; Mauri, F.; Piscanec, S.; Jiang, D.; Novoselov, K. S.; Roth, S.; Geim, A. K. Phys. Rev. Lett. 2006, $\underline{97,} 187401$.

(2) Graf, D.; Molitor, F.; Ensslin, K.; Stampfer, C.; Jungen, A.; Hierold, C.; Wirtz, L. Nano Lett. 2007, 7, 238-242.

(3) Saito, R.; Hofmann, M.; Dresselhaus, G.; Jorio, A.; Dresselhaus, M. S. Advances in

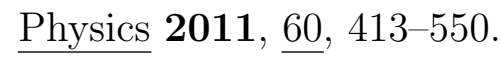

(4) Ferrari, A. C.; Basko, D. M. Nature Nanotechnology 2013, 8, 235-.

(5) Lazzeri, M.; Mauri, F. Phys. Rev. Lett. 2006, 97, 266407.

(6) Pisana, S.; Lazzeri, M.; Casiraghi, C.; Novoselov, K. S.; Geim, A. K.; Ferrari, A. C.; Mauri, F. Nature Materials 2007, 6, 198-201.

(7) Bianchi, M.; Rienks, E. D. L.; Lizzit, S.; Baraldi, A.; Balog, R.; Hornekær, L.; Hofmann, P. Phys. Rev. B 2010, 81, 041403. 
(8) Haberer, D.; Petaccia, L.; Fedorov, A. V.; Praveen, C. S.; Fabris, S.; Piccinin, S.; Vilkov, O.; Vyalikh, D. V.; Preobrajenski, A.; Verbitskiy, N. I.; Shiozawa, H.; Fink, J.; Knupfer, M.; Büchner, B.; Grüneis, A. Phys. Rev. B 2013, 88, 081401.

(9) Fedorov, A. V.; Verbitskiy, N. I.; Haberer, D.; Struzzi, C.; Petaccia, L.; Usachov, D.; Vilkov, O. Y.; Vyalikh, D. V.; Fink, J.; Knupfer, M.; Büchner, B.; Grüneis, A. Nat. Commun. 2014, 5, 4257.

(10) Verbitskiy, N. I.; Fedorov, A. V.; Tresca, C.; Profeta, G.; Petaccia, L.; Senkovskiy, B. V.; Usachov, D. Y.; Vyalikh, D. V.; Yashina, L. V.; Eliseev, A. A.; Pichler, T.; Grüneis, A. $\underline{\text { DD Materials 2016, }}$ 3, 045003.

(11) Kleeman, J.; Sugawara, K.; Sato, T.; Takahashi, T. Journal of Physics: Condensed Matter 2016, 28, 204001.

(12) Usachov, D. Y.; Fedorov, A. V.; Vilkov, O. Y.; Ogorodnikov, I. I.; Kuznetsov, M. V.; Grüneis, A.; Laubschat, C.; Vyalikh, D. V. Phys. Rev. B 2018, 97, 085132.

(13) Stampfer, C.; Molitor, F.; Graf, D.; Ensslin, K.; Jungen, A.; Hierold, C.; Wirtz, L. Applied Physics Letters 2007, 91, 241907.

(14) Das, A.; Pisana, S.; Chakraborty, B.; Piscanec, S.; Saha, S. K.; Waghmare, U. V.; Novoselov, K. S.; Krishnamurthy, H. R.; Geim, A. K.; Ferrari, A. C.; Sood, A. K. Nature Nanotechnology 2008, 3, 210-.

(15) Chen, C.-F.; Park, C.-H.; Boudouris, B. W.; Horng, J.; Geng, B.; Girit, C.; Zettl, A.; Crommie, M. F.; Segalman, R. A.; Louie, S. G.; Wang, F. Nature 2011, 471, 617-.

(16) Froehlicher, G.; Berciaud, S. Phys. Rev. B 2015, 91, 205413.

(17) Efetov, D. K.; Kim, P. Phys. Rev. Lett. 2010, 105, 256805. 
(18) Ye, J.; Craciun, M. F.; Koshino, M.; Russo, S.; Inoue, S.; Yuan, H.; Shimotani, H.; Morpurgo, A. F.; Iwasa, Y. Proceedings of the National Academy of Sciences 2011, 108, 13002-13006.

(19) Profeta, G.; Calandra, M.; Mauri, F. Nat Phys 2012, $\underline{8}, 131$.

(20) Chapman, J.; Su, Y.; Howard, C. A.; Kundys, D.; Grigorenko, A. N.; Guinea, F.; Geim, A. K.; Grigorieva, I. V.; Nair, R. R. Scientific Reports 2016, $\underline{6}, 23254-$.

(21) Ichinokura, S.; Sugawara, K.; Takayama, A.; Takahashi, T.; Hasegawa, S. ACS Nano 2016, 10, 2761-2765, PMID: 26815333.

(22) Nandkishore, R.; Levitov, L. S.; Chubukov, A. V. Nature Physics 2012, $\underline{8}, 158-$.

(23) Lifshitz, I. Soviet Physics JETP 1960, 11, 1130.

(24) McChesney, J. L.; Bostwick, A.; Ohta, T.; Seyller, T.; Horn, K.; González, J.; Rotenberg, E. Phys. Rev. Lett. 2010, 104, 136803.

(25) Mak, K. F.; da Jornada, F. H.; He, K.; Deslippe, J.; Petrone, N.; Hone, J.; Shan, J.; Louie, S. G.; Heinz, T. F. Phys. Rev. Lett. 2014, 112, 207401.

(26) Yoon, D.; Jeong, D.; Lee, H.-J.; Saito, R.; Son, Y.-W.; Lee, H. C.; Cheong, H. Carbon 2013, $\underline{61}, 373-378$.

(27) Jung, N.; Kim, B.; Crowther, A. C.; Kim, N.; Nuckolls, C.; Brus, L. ACS Nano 2011, $\underline{5}, 5708-5716$.

(28) Farhat, H.; Berciaud, S.; Kalbac, M.; Saito, R.; Heinz, T. F.; Dresselhaus, M. S.; Kong, J. Phys. Rev. Lett. 2011, 107, 157401.

(29) Ma, Y.; Kim, Y.; Kalugin, N. G.; Lombardo, A.; Ferrari, A. C.; Kono, J.; Imambekov, A.; Smirnov, D. Phys. Rev. B 2014, 89, 121402. 
(30) Hasdeo, E. H.; Nugraha, A. R. T.; Dresselhaus, M. S.; Saito, R. Phys. Rev. B 2014, $\underline{90,} 245140$.

(31) Hasdeo, E. H. Gate modulated Raman spectroscopy of graphene. Ph.D. thesis, Tohoku University, Dept. of Physics, 2016.

(32) Eklund, P. C.; Subbaswamy, K. R. Phys. Rev. B 1979, 20, 5157-5161.

(33) Dresselhaus, M. S.; Dresselhaus, G. Advances in Physics 2002, 51, 1-186.

(34) Kalbac, M.; Reina-Cecco, A.; Farhat, H.; Kong, J.; Kavan, L.; Dresselhaus, M. S. ACS Nano 2010, 4, 6055-6063, PMID: 20931995.

(35) Basko, D. M. New Journal of Physics 2009, 11, 095011.

(36) Howard, C. A.; Dean, M. P. M.; Withers, F. Phys. Rev. B 2011, 84, 241404.

(37) Parret, R.; Paillet, M.; Huntzinger, J.-R.; Nakabayashi, D.; Michel, T.; Tiberj, A.; Sauvajol, J.-L.; Zahab, A. A. ACS Nano 2013, 7, 165-173, PMID: 23194077.

(38) Zhao, W.; Tan, P. H.; Liu, J.; Ferrari, A. C. Journal of the American Chemical Society 2011, 133, 5941-5946, PMID: 21434632.

(39) Kanetani, K.; Sugawara, K.; Sato, T.; Shimizu, R.; Iwaya, K.; Hitosugi, T.; Takahashi, T. Proceedings of the National Academy of Sciences 2012, 109, 19610-19613.

(40) Ludbrook, B. M. et al. Proceedings of the National Academy of Sciences 2015, 112, $11795-11799$.

(41) Dombrowski, D.; Jolie, W.; Petrović, M.; Runte, S.; Craes, F.; Klinkhammer, J.; Kralj, M.; Lazić, P.; Sela, E.; Busse, C. Phys. Rev. Lett. 2017, 118, 116401.

(42) Starodub, E.; Bostwick, A.; Moreschini, L.; Nie, S.; Gabaly, F. E.; McCarty, K. F.; Rotenberg, E. Phys. Rev. B 2011, 83, 125428. 
(43) Busse, C.; Lazić, P.; Djemour, R.; Coraux, J.; Gerber, T.; Atodiresei, N.; Caciuc, V.; Brako, R.; N'Diaye, A. T.; Blügel, S.; Zegenhagen, J.; Michely, T. Phys. Rev. Lett. 2011, 107, 036101.

(44) Pletikosić, I.; Kralj, M.; Pervan, P.; Brako, R.; Coraux, J.; N’Diaye, A. T.; Busse, C.; Michely, T. Phys. Rev. Lett. 2009, 102, 056808.

(45) Petrovic, M. et al. Nature Communications 2013, $\underline{4}, 2772-$.

(46) Grüneis, A.; Attaccalite, C.; Wirtz, L.; Shiozawa, H.; Saito, R.; Pichler, T.; Rubio, A. Phys. Rev. B 2008, 78, 205425.

(47) Hattab, H.; NDiaye, A. T.; Wall, D.; Klein, C.; Jnawali, G.; Coraux, J.; Busse, C.; van Gastel, R.; Poelsema, B.; Michely, T.; Meyer zu Heringdorf, F.-J.; Horn-von Hoegen, M. Nano Letters 2012, 12, 678-682, PMID: 22175792.

(48) Jean, F.; Zhou, T.; Blanc, N.; Felici, R.; Coraux, J.; Renaud, G. Phys. Rev. B 2013, $\underline{88}, 165406$.

(49) Bronsgeest, M. S.; Bendiab, N.; Mathur, S.; Kimouche, A.; Johnson, H. T.; Coraux, J.; Pochet, P. Nano Letters 2015, 15, 5098-5104, PMID: 26171667.

(50) Sánchez-Barriga, J.; Varykhalov, A.; Marchenko, D.; Scholz, M. R.; Rader, O. Phys. Rev. B 2012, 85, 201413.

(51) Usachov, D. Y.; Davydov, V. Y.; Levitskii, V. S.; Shevelev, V. O.; Marchenko, D.; Senkovskiy, B. V.; Vilkov, O. Y.; Rybkin, A. G.; Yashina, L. V.; Chulkov, E. V.; Sklyadneva, I. Y.; Heid, R.; Bohnen, K.-P.; Laubschat, C.; Vyalikh, D. V. ACS Nano 2017, 11, 6336-6345, PMID: 28494148.

(52) Frank, O.; Tsoukleri, G.; Riaz, I.; Papagelis, K.; Parthenios, J.; Ferrari, A. C.; Geim, A. K.; Novoselov, K. S.; Galiotis, C. Nature Communications 2011, 2, 255- 
(53) Mueller, N. S. et al. 2D Materials 2018, 5, 015016.

(54) Pozzo, M.; Alfè, D.; Lacovig, P.; Hofmann, P.; Lizzit, S.; Baraldi, A. Phys. Rev. Lett. 2011, 106, 135501.

(55) White, G. K.; Pawlowicz, A. T. Journal of Low Temperature Physics 1970, 2, 631-639.

(56) Halvorson, J. J.; Wimber, R. T. Journal of Applied Physics 1972, 43, 2519-2522.

(57) Mohiuddin, T. M. G.; Lombardo, A.; Nair, R. R.; Bonetti, A.; Savini, G.; Jalil, R.; Bonini, N.; Basko, D. M.; Galiotis, C.; Marzari, N.; Novoselov, K. S.; Geim, A. K.; Ferrari, A. C. Phys. Rev. B 2009, 79, 205433.

(58) Cerdeira, F.; Fjeldly, T. A.; Cardona, M. Solid State Communications 1973, 13, 325 328.

(59) Tristant, D.; Wang, Y.; Gerber, I.; Monthioux, M.; Pnicaud, A.; Puech, P. Journal of Applied Physics 2015, 118, 044304.

(60) Choi, W. S.; Seo, S. S. A.; Kim, K. W.; Noh, T. W.; Kim, M. Y.; Shin, S. Phys. Rev. B 2006, 74, 205117.

(61) Lazzeri, M.; Attaccalite, C.; Wirtz, L.; Mauri, F. Phys. Rev. B 2008, 78, 081406.

(62) Nixon, D. E.; Parry, G. S. Journal of Physics C: Solid State Physics 1969, 2, 1732.

(63) Grüneis, A.; Attaccalite, C.; Rubio, A.; Vyalikh, D. V.; Molodtsov, S. L.; Fink, J.; Follath, R.; Eberhardt, W.; Büchner, B.; Pichler, T. Phys. Rev. B 2009, 80, 075431.

(64) Allard, A.; Wirtz, L. Nano Lett. 2010, 10, 4335-4340.

(65) Endlich, M.; Molina-Sánchez, A.; Wirtz, L.; Kröger, J. Phys. Rev. B 2013, 88, 205403.

(66) Ulstrup, S.; Andersen, M.; Bianchi, M.; Barreto, L.; Hammer, B.; Hornekr, L.; Hofmann, P. 2D Materials 2014, 1, 025002. 
(67) Vinogradov, N. A.; Simonov, K. A.; Generalov, A. V.; Vinogradov, A. S.; Vyalikh, D. V.; Laubschat, C.; Mrtensson, N.; Preobrajenski, A. B. Journal of Physics: Condensed Matter 2012, 24, 314202.

(68) Mialitsin, A.; Kim, J. S.; Kremer, R. K.; Blumberg, G. Phys. Rev. B 2009, 79, 064503.

(69) García-Ruiz, A.; Mucha-Kruczyński, M.; Fal'ko, V. I. Phys. Rev. B 2018, 97, 155405.

(70) Lu, H.-Y.; Chen, S.; Xu, Y.; Zhang, L.-Q.; Wang, D.; Wang, W.-S. Phys. Rev. B 2013, 88, 085416.

(71) van Gastel, R.; NDiaye, A. T.; Wall, D.; Coraux, J.; Busse, C.; Buckanie, N. M.; Meyer zu Heringdorf, F.-J.; Horn von Hoegen, M.; Michely, T.; Poelsema, B. Appl. Phys. Lett. 2009, 95, 121901-.

(72) Coraux, J.; N'Diaye, A. T.; Engler, M.; Busse, C.; Wall, D.; Buckanie, N.; zu Heringdorf, F.-J. M.; van Gastel, R.; Poelsema, B.; Michely, T. New Journal of Physics 2009, $\underline{11}, 023006$.

(73) Petaccia, L.; Vilmercati, P.; Gorovikov, S.; Barnaba, M.; Bianco, A.; Cocco, D.; Masciovecchio, C.; Goldoni, A. Nuclear Instruments and Methods in Physics Research Section A: Accelerators, Spectrometers, Detectors and Associated Equipment 2009, 606, 780-784.

(74) $\underline{\text { SPIP 6.5.1, Image Metrology }}$

(75) Grüneis, A.; Senkovskiy, B.; Fedorov, A.; Hell, M.; Michel, S. Reference Module in Chemistry, Molecular Sciences and Chemical Engineering; Elsevier, 2017; pp -.

(76) Faris, G. W.; Copeland, R. A. Appl. Opt. 1997, 36, 2684-2685.

(77) Barrett, J. J.; Adams, N. I. J. Opt. Soc. Am. 1968, 58, 311-319. 
(78) YU, P.; Cardona, M. Fundamentals of Semiconductors: Physics and Materials Properties; Graduate Texts in Physics; Springer Berlin Heidelberg, 2010.

(79) Jiang, J.; Saito, R.; Samsonidze, G. G.; Chou, S. G.; Jorio, A.; Dresselhaus, G.; Dresselhaus, M. S. Phys. Rev. B 2005, 72, 235408.

(80) Grüneis, A.; Saito, R.; Samsonidze, G. G.; Kimura, T.; Pimenta, M. A.; Jorio, A.; Filho, A. G. S.; Dresselhaus, G.; Dresselhaus, M. S. Phys. Rev. B 2003, 67, 165402.

(81) Jiang, J.; Saito, R.; Samsonidze, G. G.; Jorio, A.; Chou, S. G.; Dresselhaus, G.; Dresselhaus, M. S. Phys. Rev. B 2007, 75, 035407. 